Supporting Information for

\title{
Center-Environment Feature Model for Machine Learning Study of Spinel Oxides Based on First-Principles Computations
}

\author{
Yihang Li ${ }^{\dagger}$, Bin Xiao ${ }^{\dagger}$, Yuchao Tang $\$$, Fu Liu ${ }^{\ddagger}$, Xiaomeng Wang $\$$, Feinan Yan ${ }^{\S}$, \\ and Yi Liü, ${ }^{\dagger, *}$ \\ $\dagger$ Materials Genome Institute, Shanghai University, Shanghai, 200444, China \\ $\$$ Department of Physics, College of Science, Shanghai University, Shanghai, 200444, \\ China \\ $\S$ Department of Physics, College of Science, University of Shanghai for Science and \\ Technology, Shanghai, 200093, China \\ *Corresponding author: yiliu@shu.edu.cn (Yi Liu)
}




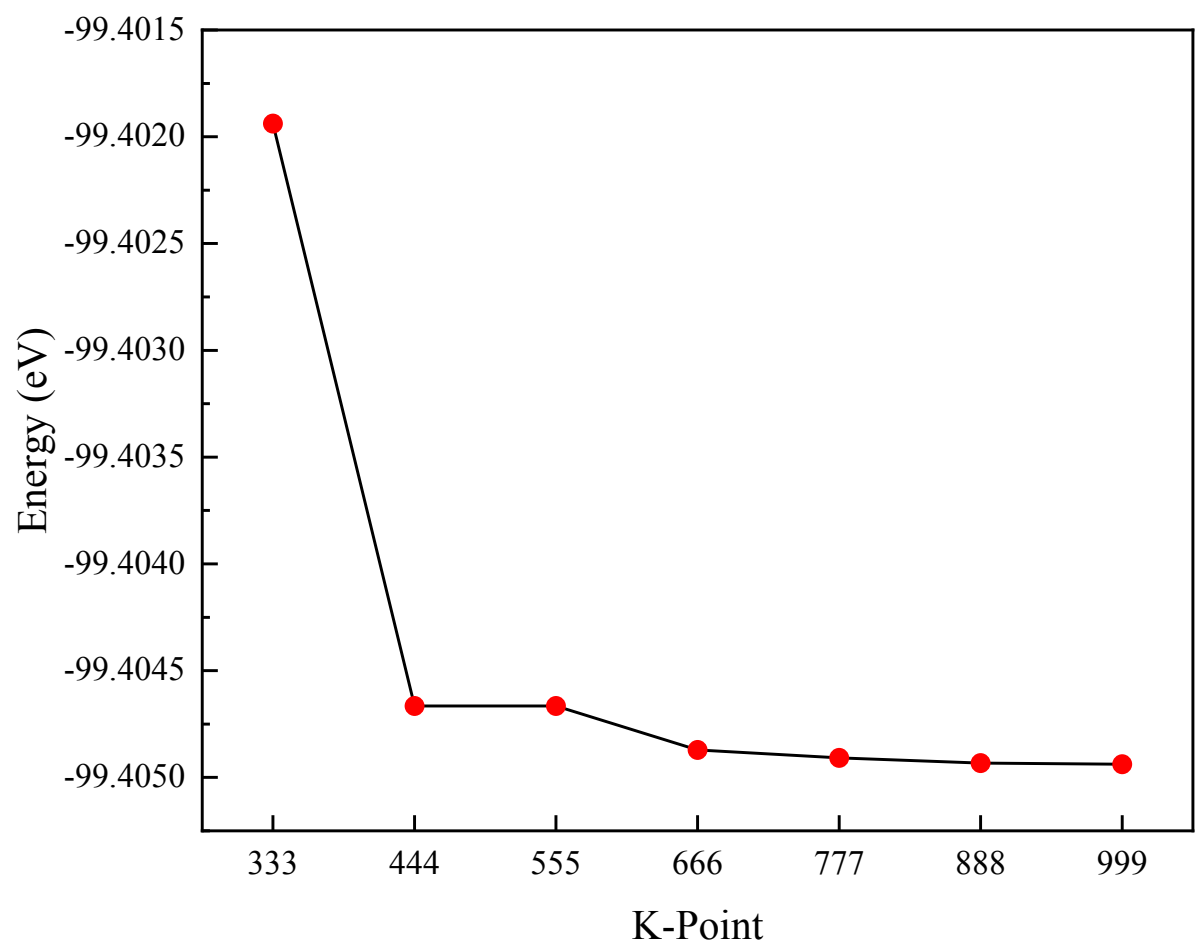

Fig. S1. According to the convergence test of k-point, integrations in the Brillouin zone were performed using $7 \times 7 \times 7 \mathrm{k}$-points grid.

Table S1. The GGA-PBE pseudo-potentials used for each element species in this study

\begin{tabular}{|c|c|c|c|c|c|c|c|c|c|c|}
\hline Li_sv & $\mathrm{Be}$ & $\mathrm{B}$ & $\mathrm{Na} \_p v$ & $\mathrm{Mg}$ & $\mathrm{Al}$ & $\mathrm{Si}$ & K_sv & $\mathrm{Ca} \_\mathrm{sv}$ & Sc_sv & Ti sv \\
\hline V_sv & $\mathrm{Cr} \_\mathrm{pv}$ & Mn_pv & $\mathrm{Fe}$ & $\mathrm{Co}$ & $\mathrm{Ni}$ & $\mathrm{Cu}$ & $\mathrm{Zn}$ & $\mathrm{a}$ & Ge_d & As \\
\hline $\mathrm{Rb} \_\mathrm{sv}$ & $\mathrm{Sr} \_\mathrm{sv}$ & Y_sv & $\mathrm{Zr} \_\mathrm{sv}$ & $\mathrm{Nb \_ sv}$ & Tc_pv & $\mathrm{Ru} \_p v$ & $\mathrm{Ru} \_p v$ & $\mathrm{Rh} \_\mathrm{pv}$ & $\mathrm{Pd}$ & $\mathrm{Ag}$ \\
\hline $\mathrm{Cd}$ & $\mathrm{I}_{1}$ & $\mathrm{Sn} \_\mathrm{d}$ & $\mathrm{Sb}$ & $\mathrm{Te}$ & $\mathrm{sv}$ & $\mathrm{Ba}$ & $\mathrm{La}$ & $\mathrm{Ce}$ & Pr_3 & $\mathrm{Nd} \_3$ \\
\hline $\mathrm{Pm} \_3$ & $\mathrm{Sm} \_3$ & u- & Gd_3 & Tb_3 & Dy_3 & Ho_3 & Er_3 & $\mathrm{Tm}_{-} 3$ & $\mathrm{Yb} \_2$ & Lu_3 \\
\hline Hf_pv & Ta_pv & W_pv & $\operatorname{Re}$ & Os & Ir & $\mathrm{Pt}$ & $\mathrm{Au}$ & $\mathrm{Hg}$ & Tl_d & $\mathrm{Pb} \_\mathrm{d}$ \\
\hline Bi_d & Ac & Th & $\mathrm{Pa}$ & II & $\mathrm{Np}$ & $\mathrm{Pu}$ & & & & \\
\hline
\end{tabular}

\section{Text S1. Some technical details for NuSVR}

Given the training sample $D=\left\{\left(X_{1}, \mathrm{Y}_{1}\right),\left(X_{2}, Y_{2}\right), \ldots,\left(X_{M}, Y_{M}\right)\right\}, Y_{i} \in R$, we hope to find a regression plane so that all the data are closest to the plane. This is called support vector regression (SVR). Suppose that we can tolerate the maximum deviation of $\varepsilon$ between $F(x)$ and $y$, that is, only when the absolute value of the difference between $F(x)$ and $y$ is greater than $\varepsilon$, then the SVR problem can be formalized as:

$$
\min _{w, b} \frac{1}{2}\|w\|^{2}+C \sum_{i=\dot{*}^{*}}^{m} l_{\varepsilon}\left(f\left(x_{i}\right), y_{i}\right)
$$

where, $C$ is the regularization constant and $l_{\varepsilon}$ is the $\epsilon$ - insensitive loss function: 


$$
l_{\varepsilon}(z)=\left\{\begin{array}{rr}
0, & |z|<\varepsilon \\
|z|-\varepsilon, & \text { otherwise }
\end{array}\right.
$$

The key codes of SVR model training in this study are as follows:

from sklearn.svm import NuSVR

nusvr $=$ NuSVR()

parameters $=\left[\left\{{ }^{\prime} C^{\prime}:[0.1,1 e 0,1 e 1,1 e 2,1 e 3]\right.\right.$, 'gamma': [0.001,0.01, 0.1, 0.5], 'kernel': ['rbf']\}] $c l f 1=$ GridSearchCV(nusvr, parameters, scoring='neg_mean_absolute_error', $c v=5)$

clf1.fit (X_train, $y_{-}$train)

param $=$ clf1.best_params_

$\operatorname{svr} 1=N u S V R\left(C=\right.$ param.get( $\left(C^{\prime \prime}\right)$, gamma=param.get("gamma"),kernel=param.get ("kernel")) svrl.fit(X_train,y_train)

$y_{\text {_train_pred }}=$ svr1.predict $\left(X \_\right.$train $)$

$y_{-}$test_pred $=$svrl.predict $\left(X \_\right.$test $)$

Table S2. Total energies at ground state of oxygen molecules and 73 elements in spinel oxides.

\begin{tabular}{cccccccccc}
\hline Element & Energy & Ele & Ene & Ele & Ene & Ele & Ene & Ele & Ene \\
\hline $\mathrm{Ac}$ & -4.0599 & $\mathrm{Cu}$ & -3.7281 & $\mathrm{Li}$ & -1.9038 & $\mathrm{Pr}$ & -4.7028 & $\mathrm{Tc}$ & -10.3507 \\
$\mathrm{Ag}$ & -2.7164 & $\mathrm{Dy}$ & -4.5350 & $\mathrm{Lu}$ & -4.4493 & $\mathrm{Pt}$ & -6.1022 & $\mathrm{Te}$ & -3.1427 \\
$\mathrm{Al}$ & -3.7493 & $\mathrm{Er}$ & -4.4981 & $\mathrm{Mg}$ & -1.5059 & $\mathrm{Pu}$ & -13.9806 & $\mathrm{Th}$ & -7.4532 \\
$\mathrm{As}$ & -4.6721 & $\mathrm{Eu}$ & -10.2529 & $\mathrm{Mn}$ & -8.9903 & $\mathrm{Rb}$ & -0.9388 & $\mathrm{Ti}$ & -7.8363 \\
$\mathrm{Au}$ & -3.2178 & $\mathrm{Fe}$ & -8.2385 & $\mathrm{Mo}$ & -10.9340 & $\mathrm{Re}$ & -12.4245 & $\mathrm{Tl}$ & -2.2278 \\
$\mathrm{~B}$ & -6.7042 & $\mathrm{Ga}$ & -2.9045 & $\mathrm{Na}$ & -1.3095 & $\mathrm{Rh}$ & -7.2570 & $\mathrm{Tm}$ & -4.4443 \\
$\mathrm{Ba}$ & -1.9083 & $\mathrm{Gd}$ & -4.5821 & $\mathrm{Nb}$ & -10.2177 & $\mathrm{Ru}$ & -9.1229 & $\mathrm{U}$ & -11.1377 \\
$\mathrm{Be}$ & -3.7649 & $\mathrm{Ge}$ & -4.5182 & $\mathrm{Nd}$ & -4.7159 & $\mathrm{Sb}$ & -4.1409 & $\mathrm{~V}$ & -8.9945 \\
$\mathrm{Bi}$ & -3.8936 & $\mathrm{Hf}$ & -9.9249 & $\mathrm{Ni}$ & -5.4681 & $\mathrm{Sc}$ & -6.2477 & $\mathrm{~W}$ & -12.9550 \\
$\mathrm{Ca}$ & -1.9255 & $\mathrm{Hg}$ & -0.1880 & $\mathrm{~Np}$ & -12.7260 & $\mathrm{Si}$ & -5.4252 & $\mathrm{Y}$ & -6.4280 \\
$\mathrm{Cd}$ & -0.7476 & $\mathrm{Ho}$ & -4.5148 & $\mathrm{Os}$ & -11.2488 & $\mathrm{Sm}$ & -4.6494 & $\mathrm{Yb}$ & -1.4652 \\
$\mathrm{Ce}$ & -5.9219 & $\mathrm{In}$ & -2.5595 & $\mathrm{~Pa}$ & -9.3966 & $\mathrm{Sn}$ & -3.8503 & $\mathrm{Zn}$ & -1.1057 \\
$\mathrm{Co}$ & -7.0181 & $\mathrm{Ir}$ & -8.8499 & $\mathrm{~Pb}$ & -3.5646 & $\mathrm{Sr}$ & -1.6384 & $\mathrm{Zr}$ & -8.5193 \\
$\mathrm{Cr}$ & -9.5128 & $\mathrm{~K}$ & -1.0510 & $\mathrm{Pd}$ & -5.2205 & $\mathrm{Ta}$ & -11.8148 & $\mathrm{O} 2$ & -9.8550 \\
$\mathrm{Cs}$ & -0.8518 & $\mathrm{La}$ & -4.8858 & $\mathrm{Pm}$ & -4.6878 & $\mathrm{~Tb}$ & -4.5583 & & \\
\hline
\end{tabular}


Table S3. Atomic and structural properties of elements and pure substances as elemental properties.

Property

\begin{tabular}{|c|c|}
\hline Atomic weight & Distance from core electron (Schubert) \\
\hline Boiling temperature & Distance from valence electron (Schubert) \\
\hline Charge nuclear effective (Clementi) & Electrochemical weight equivalent \\
\hline Compression modulus & Electronegativity (Alfred-Rochow) \\
\hline Density & Electronegativity (Martynov\&Batsanov) \\
\hline Melting temperature & Electronegativity (Pauling) \\
\hline Mendeleev chemists sequence & Electronegativity absolute \\
\hline Mendeleev d-t start left & Energy cohesive (Brewer) \\
\hline Mendeleev d-t start right & Energy of ionization first \\
\hline Mendeleev $\mathrm{H}$ d-t start left & Energy of ionization second \\
\hline Mendeleev $\mathrm{H}$ d-t start right & Energy of ionization third \\
\hline Mendeleev $\mathrm{H}$ t-d start left & Enthalpy of atomization \\
\hline Oxidation state first & Enthalpy of melting \\
\hline Quantum number & Enthalpy of vaporization \\
\hline Radii covalent & Entropy of solid \\
\hline Radii metal (Waber) & Group number \\
\hline Mendeleev $\mathrm{H}$ t-d start right & Molar heat capacity \\
\hline Mendeleev Pettifor & Moment nuclear magnetic \\
\hline Mendeleev Pettifor regular & Nuclear charge effective (Slater) \\
\hline Mendeleev t-d start left & Thermal neutron capture cross section \\
\hline Mendeleev t-d start right & Valence electron number \\
\hline Mass attenuation coefficient for MoK $\alpha$ & Radii pseudo-potential (Zunger) \\
\hline Spectral lines n0 & Magnetic resonance \\
\hline Spin nuclei & Volume of atom (Villars, Daams) \\
\hline Magnetic frequency of nuclei & Atomic electron scattering factor at 0.5 \\
\hline $\begin{array}{l}\text { Periodic number start counting top right, right-left } \\
\text { sequence }\end{array}$ & $\begin{array}{l}\text { Periodic number start counting left bottom, left- } \\
\text { right sequence }\end{array}$ \\
\hline $\begin{array}{l}\text { Atomic number start counting left top, left-right } \\
\text { sequence }\end{array}$ & Atomic environment number (Villars, Daams) \\
\hline
\end{tabular}


Table S4. Data tables of training and testing sets whose rows represent each sample of the 5329 spinel structures and columns are the features constructed based on the CE models described above plus the target values calculated by DFT.

\begin{tabular}{ccccccccc}
\hline No. & Structure & Feature1 & Feature2 & Feature3 & Feature4 & Feature5 & Feature $\mathbf{N}$ & Target \\
\hline 1 & $\mathrm{AcAc}_{2} \mathrm{O}_{4}$ & 5.084 & 17.597 & 130.850 & 331.216 & 4972.601 & $\ldots$ & -3.035 \\
2 & $\mathrm{AcAg}_{2} \mathrm{O}_{4}$ & 4.656 & 17.489 & 117.846 & 294.507 & 4638.045 & $\ldots$ & -0.962 \\
3 & $\mathrm{AcAl}_{2} \mathrm{O}_{4}$ & 4.125 & 17.500 & 107.989 & 271.061 & 4730.198 & $\ldots$ & -2.559 \\
4 & $\mathrm{AcAs}_{2} \mathrm{O}_{4}$ & 4.505 & 15.746 & 113.783 & 284.944 & 4185.670 & $\ldots$ & -1.371 \\
5 & $\mathrm{AcAu}_{2} \mathrm{O}_{4}$ & 4.965 & 17.491 & 127.163 & 320.446 & 4826.720 & $\ldots$ & -0.783 \\
6 & $\mathrm{AcB}_{2} \mathrm{O}_{4}$ & 4.079 & 14.295 & 105.664 & 266.367 & 5078.612 & $\ldots$ & -0.837 \\
7 & $\mathrm{AcBa}_{2} \mathrm{O}_{4}$ & 4.766 & 18.181 & 120.800 & 303.913 & 4498.245 & $\ldots$ & -2.332 \\
8 & $\mathrm{AcBe}_{2} \mathrm{O}_{4}$ & 4.068 & 17.501 & 105.367 & 265.826 & 4876.953 & $\ldots$ & -1.926 \\
9 & $\mathrm{AcBi}_{2} \mathrm{O}_{4}$ & 5.029 & 15.786 & 129.040 & 325.771 & 4478.628 & $\ldots$ & -1.622 \\
$\mathrm{k}$ & $\ldots$ & $\ldots$ & $\ldots$ & $\ldots$ & $\ldots$ & $\ldots$ & $\ldots$ & $\ldots$ \\
5320 & $\mathrm{ZrTi}_{2} \mathrm{O}_{4}$ & 2.583 & 17.589 & 55.928 & 125.546 & 5416.670 & $\ldots$ & -2.645 \\
5321 & $\mathrm{ZrTl}_{2} \mathrm{O}_{4}$ & 3.205 & 17.559 & 73.534 & 172.270 & 4867.358 & $\ldots$ & -1.573 \\
5322 & $\mathrm{ZrTm}_{2} \mathrm{O}_{4}$ & 3.095 & 17.578 & 70.035 & 161.889 & 4953.292 & $\ldots$ & -3.022 \\
5323 & $\mathrm{ZrU}_{2} \mathrm{O}_{4}$ & 3.307 & 15.771 & 76.905 & 182.544 & 5556.518 & $\ldots$ & -2.521 \\
5324 & $\mathrm{ZrV}_{2} \mathrm{O}_{4}$ & 2.601 & 14.451 & 56.367 & 126.796 & 5352.275 & $\ldots$ & -2.242 \\
5325 & $\mathrm{ZrW}_{2} \mathrm{O}_{4}$ & 3.148 & 197.737 & 71.613 & 166.531 & 6144.992 & $\ldots$ & -1.273 \\
5326 & $\mathrm{ZrY}_{2} \mathrm{O}_{4}$ & 2.785 & 17.574 & 61.005 & 137.805 & 5312.543 & $\ldots$ & -3.012 \\
5327 & $\mathrm{ZrYb}_{2} \mathrm{O}_{4}$ & 3.111 & 17.612 & 70.376 & 163.197 & 4807.231 & $\ldots$ & -3.385 \\
5328 & $\mathrm{ZrZn}_{2} \mathrm{O}_{4}$ & 2.688 & 17.623 & 58.456 & 131.106 & 4724.398 & $\ldots$ & -2.144 \\
5329 & $\mathrm{ZrZr}_{2} \mathrm{O}_{4}$ & 2.803 & 17.614 & 61.423 & 138.777 & 5521.554 & $\ldots$ & -2.662 \\
\hline
\end{tabular}

Table S5. Formation energies of spinel oxides calculated by GGA and GGA + U methods

\begin{tabular}{|c|c|c|c|c|c|}
\hline \multirow{2}{*}{$\begin{array}{l}\text { Structure } \\
\mathrm{CoFe}_{2} \mathrm{O}_{4}\end{array}$} & \multirow{2}{*}{ 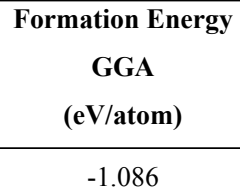 } & $\begin{array}{c}\text { Formation Energy } \\
\text { GGA+U } \\
\text { (eV/atom) }\end{array}$ & \multirow{2}{*}{$\begin{array}{c}\text { Difference } \\
-0.815\end{array}$} & \multicolumn{2}{|c|}{ U Values (eV) } \\
\hline & & -1.901 & & Co: 3.32 & Fe: 5.3 \\
\hline $\mathrm{NiFe}_{2} \mathrm{O}_{4}$ & -1.070 & -1.880 & -0.810 & Ni: 6.2 & Fe: 5.3 \\
\hline $\mathrm{MnFe}_{2} \mathrm{O}_{4}$ & -1.109 & -2.338 & -1.229 & Mn: 3.9 & Fe: 5.3 \\
\hline $\mathrm{MnV}_{2} \mathrm{O}_{4}$ & -1.962 & -2.009 & -0.047 & Mn: 3.9 & $V: 3.25$ \\
\hline $\mathrm{NiMn}_{2} \mathrm{O}_{4}$ & -1.413 & -2.080 & -0.667 & Ni: 6.2 & Mn: 3.9 \\
\hline $\mathrm{FeV}_{2} \mathrm{O}_{4}$ & -1.909 & -2.586 & -0.677 & Fe: 5.3 & $V: 3.25$ \\
\hline $\mathrm{NiCr}_{2} \mathrm{O}_{4}$ & -1.612 & -2.659 & -1.047 & Ni: 6.2 & Cr: 3.7 \\
\hline $\mathrm{CoV}_{2} \mathrm{O}_{4}$ & -1.749 & -2.517 & -0.768 & Co: 3.32 & $V: 3.25$ \\
\hline $\mathrm{MnCr}_{2} \mathrm{O}_{4}$ & -1.759 & -3.132 & -1.373 & Mn: 3.9 & Cr: 3.7 \\
\hline $\mathrm{FeCr}_{2} \mathrm{O}_{4}$ & -1.306 & -3.015 & -1.709 & Fe: 5.3 & Cr: 3.7 \\
\hline $\mathrm{NiCo}_{2} \mathrm{O}_{4}$ & -0.971 & -1.195 & -0.224 & Ni: 6.2 & Co: 3.32 \\
\hline $\mathrm{MnCo}_{2} \mathrm{O}_{4}$ & -1.022 & -1.267 & -0.245 & Mn: 3.9 & Co: 3.32 \\
\hline
\end{tabular}




\section{Text S2.}

The formation energies of all compounds are adjusted according to the modified molecular energy of $\mathrm{O}_{2}$. Data files can be obtained from this website: https://github.com/liyihang1024/ML-CE-Spinel

To confirm further stability of the predicted spinel oxides, we calculated the energies of 465 experimental oxides that are possible decomposition products of $82 \mathrm{AB}_{2} \mathrm{O}_{4}$ spinel oxides synthesized experimentally. Then we made comparisons between reactants and products of the following decomposition reactions.

$$
\begin{gathered}
\mathrm{AB}_{2} \mathrm{O}_{4} \rightarrow \mathrm{AO}+\mathrm{B}_{2} \mathrm{O}_{3} \\
\mathrm{AB}_{2} \mathrm{O}_{4} \rightarrow \mathrm{BO}+\mathrm{ABO}_{3} \\
\mathrm{AB}_{2} \mathrm{O}_{4} \rightarrow 2 \mathrm{BO}+\mathrm{AO}_{2} \\
\mathrm{AB}_{2} \mathrm{O}_{4} \rightarrow \mathrm{BO}_{2}+\mathrm{ABO}_{2}
\end{gathered}
$$

We calculated the formation energies of some spinel with reference to the most stable binary oxides, as shown in Table S6. The formation energy is defined as follows:

$$
E_{f}=E_{A B_{2} O_{4}}-E_{A_{x} O_{y}}-E_{B_{x} O_{y}}
$$

where $E_{f}$ and $E_{A B_{2} O_{4}}$ are the formation energy and total energy of spinel oxides, respectively. $E_{A_{x} O_{y}}$ and $E_{B_{x} O_{y}}$ represent the energies of the most stable binary oxides $A_{x} O_{y}$ and $B_{x} O_{y}$, respectively. The reaction energies show that 43 out of 82 "stable" spinel oxide structures are more stable than their decomposed oxides at least following one of the above reactions. These reaction energy results confirmed more than half of the predicted "stable" structures are thermodynamically more stable than the simpler counterparts and the others are possibly metastable. It implies that the predicted "more stable" structures are more likely to be thermodynamically stable or metastable. The formation energy results gave the initial quick assessment on stability. Further confirmation on decomposition reaction energies are necessary before experiment

\begin{tabular}{|c|c|c|c|c|c|c|c|c|c|c|c|c|}
\hline Structure & $\mathrm{AB}_{2} \mathrm{O}_{4}$ & $\mathrm{AO}$ & $\mathrm{B}_{2} \mathrm{O}_{3}$ & $\begin{array}{c}\mathrm{AO}+ \\
\mathrm{B}_{2} \mathrm{O}_{3} \\
-\mathrm{AB}_{2} \mathrm{O}_{4}\end{array}$ & $\mathrm{BO}$ & $\mathrm{ABO}_{3}$ & $\begin{array}{c}\mathrm{BO}+ \\
\mathrm{ABO}_{3} \\
-\mathrm{AB}_{2} \mathrm{O}_{4}\end{array}$ & $\mathrm{AO}_{2}$ & $\begin{array}{c}2 \mathrm{BO}+ \\
\mathrm{AO}_{2} \\
-\mathrm{AB}_{2} \mathrm{O}_{4}\end{array}$ & $\mathrm{BO}_{2}$ & $\mathrm{ABO}_{2}$ & $\begin{array}{c}\mathrm{BO}_{2}+ \\
\mathrm{ABO}_{2} \\
-\mathrm{AB}_{2} \mathrm{O}_{4}\end{array}$ \\
\hline $\mathrm{FeFe}_{2} \mathrm{O}_{4}$ & -47.20 & -13.44 & -33.75 & 0.02 & -13.44 & -33.75 & 0.02 & -18.44 & 1.88 & -18.44 & -26.88 & 1.88 \\
\hline $\mathrm{ZnFe}_{2} \mathrm{O}_{4}$ & -42.95 & -9.10 & -33.75 & 0.10 & -13.44 & -27.35 & 2.16 & -13.40 & 2.67 & -18.44 & -22.01 & 2.49 \\
\hline $\mathrm{CoFe}_{2} \mathrm{O}_{4}$ & -45.97 & -12.30 & -33.75 & -0.07 & -13.44 & & & -17.23 & 1.86 & -18.44 & -22.67 & 4.86 \\
\hline $\mathrm{NiFe}_{2} \mathrm{O}_{4}$ & -43.27 & -9.94 & -33.75 & -0.41 & -13.44 & -28.64 & 1.20 & -13.82 & 2.57 & -18.44 & & \\
\hline $\mathrm{MgAl}_{2} \mathrm{O}_{4}$ & -49.76 & -11.97 & -37.41 & 0.38 & -11.11 & -30.85 & 7.80 & -16.63 & 10.91 & -20.28 & & \\
\hline $\mathrm{MgFe}_{2} \mathrm{O}_{4}$ & -45.78 & -11.97 & -33.75 & 0.07 & -13.44 & -30.68 & 1.67 & -16.63 & 2.28 & -18.44 & -24.52 & 2.81 \\
\hline
\end{tabular}
synthesis.

Table S6. Formation energies of spinel oxides and corresponding binary oxides calculated by DFT. The structures in Bold have all positive reaction energies. The structures in Italic have at least one negative reaction energies. 


\begin{tabular}{|c|c|c|c|c|c|c|c|c|c|c|c|c|}
\hline $\mathrm{LiMn}_{2} \mathrm{O}_{4}$ & -48.09 & & -37.84 & & -15.71 & & & -14.54 & 2.13 & -21.43 & -26.56 & 0.09 \\
\hline $\mathrm{MnFe}_{2} \mathrm{O}_{4}$ & -49.82 & -15.71 & -33.75 & 0.36 & -13.44 & -35.74 & 0.65 & -21.43 & 1.51 & -18.44 & -27.97 & 3.40 \\
\hline $\mathrm{CuFe}_{2} \mathrm{O}_{4}$ & -43.42 & -10.25 & -33.75 & -0.57 & -13.44 & & & -14.21 & 2.34 & -18.44 & -24.12 & 0.86 \\
\hline $\mathrm{CoCo}_{2} \mathrm{O}_{4}$ & -42.02 & -12.30 & -27.82 & 1.90 & -12.30 & -27.82 & 1.90 & -17.23 & 0.19 & -17.23 & -24.60 & 0.19 \\
\hline $\mathrm{MnV}_{2} \mathrm{O}_{4}$ & -50.11 & -15.71 & -40.18 & -5.78 & -15.83 & -39.08 & -4.80 & -21.43 & -2.98 & -23.35 & & \\
\hline $\mathrm{ZnCr}_{2} \mathrm{O}_{4}$ & -49.55 & -9.10 & -39.92 & 0.53 & -15.85 & -30.89 & 2.81 & -13.40 & 4.45 & -22.32 & -24.38 & 2.86 \\
\hline $\mathrm{CoAl}_{2} \mathrm{O}_{4}$ & -50.07 & -12.30 & -37.41 & 0.37 & -11.11 & -33.57 & 5.39 & -17.23 & 10.62 & -20.28 & & \\
\hline $\mathrm{ZnAl}_{2} \mathrm{O}_{4}$ & -46.94 & -9.10 & -37.41 & 0.43 & -11.11 & & & -13.40 & 11.32 & -20.28 & & \\
\hline $\mathrm{NiMn}_{2} \mathrm{O}_{4}$ & -46.32 & -9.94 & -37.84 & -1.45 & -15.71 & -31.61 & -1.00 & -13.82 & 1.08 & -21.43 & -25.37 & -0.48 \\
\hline $\mathrm{MgCr}_{2} \mathrm{O}_{4}$ & -52.38 & -11.97 & -39.92 & 0.49 & -15.85 & -33.28 & 3.26 & -16.63 & 4.06 & -22.32 & -27.49 & 2.58 \\
\hline $\mathrm{FeV}_{2} \mathrm{O}_{4}$ & -53.32 & -13.44 & -40.18 & -0.30 & -15.83 & & & -18.44 & 3.22 & -23.35 & & \\
\hline $\mathrm{FeAl}_{2} \mathrm{O}_{4}$ & -51.07 & -13.44 & -37.41 & 0.23 & -11.11 & -35.41 & 4.55 & -18.44 & 10.41 & -20.28 & & \\
\hline $\mathrm{CuCr} \mathrm{O}_{4}$ & -50.01 & -10.25 & -39.92 & -0.16 & -15.85 & -29.66 & 4.51 & -14.21 & 4.10 & -22.32 & -27.41 & 0.28 \\
\hline $\mathrm{ZnV}_{2} \mathrm{O}_{4}$ & -48.95 & -9.10 & -40.18 & -0.33 & -15.83 & -32.05 & 1.08 & -13.40 & 3.90 & -23.35 & -24.59 & 1.02 \\
\hline $\mathrm{CdFe}_{2} \mathrm{O}_{4}$ & -41.75 & -7.91 & -33.75 & 0.09 & -13.44 & -26.54 & 1.78 & -12.45 & 2.43 & -18.44 & & \\
\hline $\mathrm{CuMn}_{2} \mathrm{O}_{4}$ & -47.79 & -10.25 & -37.84 & -0.30 & -15.71 & -31.51 & 0.56 & -14.21 & 2.15 & -21.43 & -26.27 & 0.08 \\
\hline $\mathrm{NiCr}_{2} \mathrm{O}_{4}$ & -49.55 & -9.94 & -39.92 & -0.31 & -15.85 & & & -13.82 & 4.03 & -22.32 & & \\
\hline $\mathrm{CoV}_{2} \mathrm{O}_{4}$ & -52.87 & -12.30 & -40.18 & 0.39 & -15.83 & -35.25 & 1.80 & -17.23 & 3.99 & -23.35 & & \\
\hline $\mathrm{ZnGa}_{2} \mathrm{O}_{4}$ & -39.60 & -9.10 & -30.18 & 0.31 & -10.06 & -22.81 & 6.73 & -13.40 & 6.08 & -16.29 & & \\
\hline $\mathrm{NiAl}_{2} \mathrm{O}_{4}$ & -47.23 & -9.94 & -37.41 & -0.12 & -11.11 & -29.13 & 6.99 & -13.82 & 11.19 & -20.28 & & \\
\hline $\mathrm{MgMn}_{2} \mathrm{O}_{4}$ & -48.32 & -11.97 & -37.84 & -1.48 & -15.71 & -33.74 & -1.13 & -16.63 & 0.27 & -21.43 & -27.63 & -0.74 \\
\hline $\mathrm{LiV}_{2} \mathrm{O}_{4}$ & -51.09 & & -40.18 & & -15.83 & -34.21 & 1.06 & -14.54 & 4.90 & -23.35 & -27.79 & -0.04 \\
\hline $\mathrm{SiFe}_{2} \mathrm{O}_{4}$ & -50.74 & -13.78 & -33.75 & 3.21 & -13.44 & -37.30 & 0.00 & -23.75 & 0.11 & -18.44 & & \\
\hline $\mathrm{MnCr}_{2} \mathrm{O}_{4}$ & -56.21 & -15.71 & -39.92 & 0.57 & -15.85 & & & -21.43 & 3.07 & -22.32 & & \\
\hline $\mathrm{FeCr}_{2} \mathrm{O}_{4}$ & -53.81 & -13.44 & -39.92 & 0.45 & -15.85 & -36.79 & 1.17 & -18.44 & 3.67 & -22.32 & & \\
\hline $\mathrm{MgV}_{2} \mathrm{O}_{4}$ & -51.78 & -11.97 & -40.18 & -0.37 & -15.83 & -35.64 & 0.31 & -16.63 & 3.50 & -23.35 & -26.82 & 1.61 \\
\hline $\mathrm{MgGa}_{2} \mathrm{O}_{4}$ & -42.41 & -11.97 & -30.18 & 0.26 & -10.06 & & & -16.63 & 5.67 & -16.29 & & \\
\hline $\mathrm{ZnMn}_{2} \mathrm{O}_{4}$ & -44.97 & -9.10 & -37.84 & -1.97 & -15.71 & -30.60 & -1.35 & -13.40 & 0.14 & -21.43 & -24.26 & -0.72 \\
\hline $\mathrm{CuRh} \mathrm{O}_{2} \mathrm{O}_{4}$ & -43.20 & -10.25 & -32.87 & 0.09 & & & & -14.21 & & -19.54 & -23.83 & -0.17 \\
\hline $\mathrm{SiMg}_{2} \mathrm{O}_{4}$ & -47.52 & -13.78 & & & -11.97 & -35.96 & -0.40 & -23.75 & -0.16 & -16.63 & & \\
\hline $\mathrm{SiNi}_{2} \mathrm{O}_{4}$ & -43.56 & -13.78 & -22.46 & 7.32 & -9.94 & & & -23.75 & -0.06 & -13.82 & & \\
\hline $\mathrm{CuAl}_{2} \mathrm{O}_{4}$ & -47.21 & -10.25 & -37.41 & -0.44 & -11.11 & -30.91 & 5.19 & -14.21 & 10.78 & -20.28 & -26.04 & 0.89 \\
\hline $\mathrm{CdCr}_{2} \mathrm{O}_{4}$ & -48.30 & -7.91 & -39.92 & 0.47 & -15.85 & & & -12.45 & 4.15 & -22.32 & & \\
\hline $\mathrm{ZnCo}_{2} \mathrm{O}_{4}$ & -39.17 & -9.10 & -27.82 & 2.25 & -12.30 & -26.05 & 0.82 & -13.40 & 1.17 & -17.23 & -20.99 & 0.94 \\
\hline $\mathrm{MnTi}_{2} \mathrm{O}_{4}$ & -60.81 & -15.71 & -45.09 & 0.01 & -17.97 & -42.95 & -0.10 & -21.43 & 3.44 & -26.92 & & 0.29 \\
\hline $\mathrm{LiNi}_{2} \mathrm{O}_{4}$ & -33.77 & & -22.46 & & -9.94 & -21.46 & 2.37 & -14.54 & -0.64 & -13.82 & -19.66 & 2.61 \\
\hline $\mathrm{SnZn}_{2} \mathrm{O}_{4}$ & -36.24 & -11.53 & & & -9.10 & -27.75 & -0.61 & & & -13.40 & -20.23 & \\
\hline $\mathrm{CoGa}_{2} \mathrm{O}_{4}$ & -42.75 & -12.30 & -30.18 & 0.27 & -10.06 & & & -17.23 & 5.40 & -16.29 & & \\
\hline $\mathrm{MgIn}_{2} \mathrm{O}_{4}$ & -40.05 & -11.97 & -28.27 & -0.19 & -9.72 & -24.23 & 6.09 & -16.63 & 3.98 & -15.58 & & 0.08 \\
\hline $\mathrm{NiCo}_{2} \mathrm{O}_{4}$ & -38.55 & -9.94 & -27.82 & 0.80 & -12.30 & -27.20 & -0.95 & -13.82 & 0.13 & -17.23 & -21.24 & \\
\hline $\mathrm{MoAg}_{2} \mathrm{O}_{4}$ & -39.13 & & -20.24 & & -7.98 & & & -22.22 & 0.95 & -12.44 & & \\
\hline $\mathrm{MoNa}_{2} \mathrm{O}_{4}$ & -42.42 & & & & & -31.84 & & -22.22 & & & -25.06 & 0.30 \\
\hline $\mathrm{LiTi}_{2} \mathrm{O}_{4}$ & -57.47 & & -45.09 & & -17.97 & & & -14.54 & 6.98 & -26.92 & -30.24 & \\
\hline $\mathrm{HgCr}_{2} \mathrm{O}_{4}$ & -45.86 & -5.84 & -39.92 & 0.10 & -15.85 & & & -10.08 & 4.09 & -22.32 & & \\
\hline
\end{tabular}




\begin{tabular}{|c|c|c|c|c|c|c|c|c|c|c|c|c|}
\hline $\mathrm{GeCo}_{2} \mathrm{O}_{4}$ & -44.11 & & -27.82 & & -12.30 & -31.43 & 0.39 & -19.38 & 0.14 & -17.23 & & 3.34 \\
\hline $\mathrm{SnMg}_{2} \mathrm{O}_{4}$ & -42.76 & -11.53 & & & -11.97 & -30.89 & -0.09 & & & -16.63 & -22.79 & \\
\hline $\mathrm{MnAl}_{2} \mathbf{O}_{4}$ & -53.36 & -15.71 & -37.41 & 0.24 & -11.11 & -37.39 & 4.86 & -21.43 & 9.71 & -20.28 & & \\
\hline $\mathrm{CdIn}_{2} \mathrm{O}_{4}$ & -36.41 & -7.91 & -28.27 & 0.23 & -9.72 & & & -12.45 & 4.52 & -15.58 & & \\
\hline $\mathrm{ZnIn}_{2} \mathrm{O}_{4}$ & -37.17 & -9.10 & -28.27 & -0.21 & -9.72 & & & -13.40 & 4.32 & -15.58 & & \\
\hline $\mathrm{GeNi}_{2} \mathrm{O}_{4}$ & -39.78 & & -22.46 & & -9.94 & & & -19.38 & 0.53 & -13.82 & & \\
\hline $\mathrm{MnIn}_{2} \mathrm{O}_{4}$ & -44.03 & -15.71 & -28.27 & 0.05 & -9.72 & -32.98 & 1.33 & -21.43 & 3.16 & -15.58 & & \\
\hline $\mathrm{CoRh}_{2} \mathrm{O}_{4}$ & -45.42 & -12.30 & -32.87 & 0.25 & & -27.86 & & -17.23 & & -19.54 & & \\
\hline $\mathrm{CdV}_{2} \mathrm{O}_{4}$ & -48.42 & -7.91 & -40.18 & 0.32 & -15.83 & -31.48 & 1.11 & -12.45 & 4.32 & -23.35 & & \\
\hline $\mathrm{CdRh}_{2} \mathrm{O}_{4}$ & -41.19 & -7.91 & -32.87 & 0.41 & & -25.34 & & -12.45 & & -19.54 & & \\
\hline $\mathrm{ZnRh}_{2} \mathbf{O}_{4}$ & -42.14 & -9.10 & -32.87 & 0.17 & & -24.83 & & -13.40 & & -19.54 & & \\
\hline $\mathrm{MnCo}_{2} \mathrm{O}_{4}$ & -42.40 & -15.71 & -27.82 & -1.13 & -12.30 & -34.02 & -3.92 & -21.43 & -3.63 & -17.23 & & \\
\hline $\mathrm{MgRh}_{2} \mathrm{O}_{4}$ & -44.89 & -11.97 & -32.87 & 0.05 & & -27.93 & & -16.63 & & -19.54 & & 1.26 \\
\hline $\mathrm{CuGa} a_{2} \mathrm{O}_{4}$ & -39.90 & -10.25 & -30.18 & -0.53 & -10.06 & & & -14.21 & 5.57 & -16.29 & -22.35 & \\
\hline $\mathrm{AlCo}_{2} \mathrm{O}_{4}$ & -44.48 & -11.11 & -27.82 & 5.55 & -12.30 & -33.57 & -1.39 & -20.28 & -0.40 & -17.23 & & \\
\hline $\mathrm{NiGa}_{2} \mathrm{O}_{4}$ & -36.59 & -9.94 & -30.18 & -3.53 & -10.06 & -33.57 & -7.05 & -13.82 & 2.65 & -16.29 & & \\
\hline $\mathrm{MnRh}_{2} \mathrm{O}_{4}$ & -48.91 & -15.71 & -32.87 & 0.32 & & & & -21.43 & & -19.54 & & 0.72 \\
\hline $\mathrm{MgTi}_{2} \mathrm{O}_{4}$ & -56.98 & -11.97 & -45.09 & -0.07 & -17.97 & -39.12 & -0.11 & -16.63 & 4.41 & -26.92 & -29.34 & \\
\hline $\mathrm{NiRh}_{2} \mathrm{O}_{4}$ & -41.56 & -9.94 & -32.87 & -1.24 & & & & -13.82 & & -19.54 & & \\
\hline $\mathrm{CdGa}_{2} \mathrm{O}_{4}$ & -38.27 & -7.91 & -30.18 & 0.18 & -10.06 & & & -12.45 & 5.71 & -16.29 & & \\
\hline $\mathrm{AlV}_{2} \mathrm{O}_{4}$ & -54.51 & -11.11 & -40.18 & 3.22 & -15.83 & -38.72 & -0.04 & -20.28 & 2.57 & -23.35 & & \\
\hline $\mathrm{WNa}_{2} \mathrm{O}_{4}$ & -43.75 & & & & & -33.18 & & -22.87 & & & -23.58 & 1.67 \\
\hline $\mathrm{MgCo}_{2} \mathrm{O}_{4}$ & -42.51 & -11.97 & -27.82 & 2.73 & -12.30 & -29.04 & 1.17 & -16.63 & 1.28 & -17.23 & -23.61 & \\
\hline $\mathrm{GeMg}_{2} \mathrm{O}_{4}$ & -43.97 & & & & -11.97 & -31.81 & 0.20 & -19.38 & 0.66 & -16.63 & & \\
\hline $\mathrm{SiCo}_{2} \mathrm{O}_{4}$ & -47.92 & -13.78 & -27.82 & 6.32 & -12.30 & -35.96 & -0.34 & -23.75 & -0.43 & -17.23 & & \\
\hline $\mathrm{SnCd}_{2} \mathrm{O}_{4}$ & -34.24 & -11.53 & & & -7.91 & -26.94 & -0.62 & & & -12.45 & & \\
\hline $\mathrm{PdZn}_{2} \mathrm{O}_{4}$ & -32.14 & & & & -9.10 & & & -15.88 & -1.95 & -13.40 & & \\
\hline $\mathrm{AlMn}_{2} \mathrm{O}_{4}$ & -52.77 & -11.11 & -37.84 & 3.82 & -15.71 & -37.39 & -0.33 & -20.28 & 1.07 & -21.43 & & \\
\hline $\mathrm{GeFe}_{2} \mathrm{O}_{4}$ & -46.91 & & -33.75 & & -13.44 & -30.85 & 2.63 & -19.38 & 0.66 & -18.44 & & \\
\hline $\mathrm{AlFe}_{2} \mathrm{O}_{4}$ & -48.74 & -11.11 & -33.75 & 3.88 & -13.44 & -35.41 & -0.11 & -20.28 & 1.58 & -18.44 & & 1.27 \\
\hline $\mathrm{CuCo}_{2} \mathrm{O}_{4}$ & -40.18 & -10.25 & -27.82 & 2.11 & -12.30 & & & -14.21 & 1.37 & -17.23 & -21.68 & \\
\hline $\mathrm{CdAl}_{2} \mathrm{O}_{4}$ & -45.24 & -7.91 & -37.41 & -0.08 & -11.11 & & & -12.45 & 10.57 & -20.28 & & \\
\hline
\end{tabular}


Table S7. Test Set $\mathrm{R}^{2}$ and RMSE of machine learning results for each model of total energy

\begin{tabular}{ccccccccccc}
\hline $\mathbf{R}^{2}$ & $\mathbf{1}$ & $\mathbf{2}$ & $\mathbf{3}$ & $\mathbf{4}$ & $\mathbf{5}$ & $\mathbf{6}$ & $\mathbf{7}$ & $\mathbf{8}$ & $\mathbf{9}$ & $\mathbf{1 0}$ \\
\hline CE-1 & 0.153 & 0.959 & 0.961 & 0.960 & 0.959 & 0.959 & 0.959 & 0.960 & 0.961 & 0.958 \\
$\mathbf{C E - 2}$ & 0.993 & 0.995 & 0.996 & 0.995 & 0.996 & 0.996 & 0.995 & 0.996 & 0.996 & 0.996 \\
CE-3 & 0.782 & 0.786 & 0.780 & 0.984 & 0.983 & 0.983 & 0.981 & 0.978 & 0.983 & 0.982 \\
CE-4 & 0.843 & 0.995 & 0.995 & 0.992 & 0.992 & 0.992 & 0.992 & 0.993 & 0.992 & 0.992 \\
CE-5 & 0.842 & 0.991 & 0.992 & 0.977 & 0.974 & 0.968 & 0.982 & 0.988 & 0.974 & 0.979 \\
CE-6 & 0.150 & 0.994 & 0.994 & 0.994 & 0.994 & 0.993 & 0.995 & 0.995 & 0.993 & 0.993 \\
CE-7 & 0.785 & 0.783 & 0.784 & 0.995 & 0.994 & 0.995 & 0.994 & 0.992 & 0.994 & 0.993 \\
CE-8 & 0.994 & 0.996 & 0.996 & 0.995 & 0.995 & 0.996 & 0.996 & 0.996 & 0.996 & 0.996 \\
\hline \hline RMSE & $\mathbf{1}$ & $\mathbf{2}$ & $\mathbf{3}$ & $\mathbf{4}$ & $\mathbf{5}$ & $\mathbf{6}$ & $\mathbf{7}$ & $\mathbf{8}$ & $\mathbf{9}$ & $\mathbf{1 0}$ \\
\hline CE-1 & 1.315 & 0.287 & 0.283 & 0.284 & 0.286 & 0.288 & 0.287 & 0.287 & 0.282 & 0.291 \\
CE-2 & 0.116 & 0.097 & 0.090 & 0.096 & 0.093 & 0.094 & 0.097 & 0.093 & 0.090 & 0.093 \\
CE-3 & 0.666 & 0.662 & 0.667 & 0.177 & 0.185 & 0.189 & 0.196 & 0.209 & 0.182 & 0.190 \\
CE-4 & 0.562 & 0.101 & 0.101 & 0.127 & 0.130 & 0.125 & 0.124 & 0.115 & 0.125 & 0.126 \\
CE-5 & 0.565 & 0.136 & 0.131 & 0.215 & 0.227 & 0.253 & 0.191 & 0.157 & 0.228 & 0.208 \\
CE-6 & 1.310 & 0.109 & 0.109 & 0.110 & 0.106 & 0.117 & 0.104 & 0.104 & 0.119 & 0.117 \\
CE-7 & 0.664 & 0.661 & 0.659 & 0.103 & 0.106 & 0.103 & 0.106 & 0.125 & 0.108 & 0.120 \\
CE-8 & 0.108 & 0.089 & 0.089 & 0.097 & 0.097 & 0.095 & 0.093 & 0.090 & 0.096 & 0.094 \\
\hline
\end{tabular}

Table S8. Test Set $\mathrm{R}^{2}$ and RMSE of machine learning results for each model of band gap.

\begin{tabular}{ccccccccccc}
\hline $\mathbf{R}^{\mathbf{2}}$ & $\mathbf{1}$ & $\mathbf{2}$ & $\mathbf{3}$ & $\mathbf{4}$ & $\mathbf{5}$ & $\mathbf{6}$ & $\mathbf{7}$ & $\mathbf{8}$ & $\mathbf{9}$ & $\mathbf{1 0}$ \\
\hline $\mathbf{C E}-1$ & 0.118 & 0.567 & 0.700 & 0.672 & 0.689 & 0.690 & 0.703 & 0.709 & 0.679 & 0.679 \\
$\mathbf{C E - 2}$ & 0.802 & 0.783 & 0.803 & 0.811 & 0.813 & 0.783 & 0.784 & 0.810 & 0.795 & 0.806 \\
$\mathbf{C E - 3}$ & 0.226 & 0.250 & 0.444 & 0.667 & 0.640 & 0.617 & 0.625 & 0.544 & 0.666 & 0.644 \\
$\mathbf{C E - 4}$ & 0.628 & 0.786 & 0.763 & 0.785 & 0.791 & 0.776 & 0.798 & 0.753 & 0.793 & 0.773 \\
CE-5 & 0.723 & 0.722 & 0.761 & 0.768 & 0.732 & 0.735 & 0.740 & 0.747 & 0.738 & 0.713 \\
CE-6 & 0.133 & 0.553 & 0.802 & 0.809 & 0.794 & 0.806 & 0.814 & 0.780 & 0.798 & 0.807 \\
CE-7 & 0.183 & 0.267 & 0.527 & 0.795 & 0.773 & 0.799 & 0.806 & 0.740 & 0.751 & 0.719 \\
CE-8 & 0.626 & 0.770 & 0.775 & 0.795 & 0.779 & 0.788 & 0.806 & 0.782 & 0.805 & 0.820 \\
\hline \hline RMSE & $\mathbf{1}$ & $\mathbf{2}$ & $\mathbf{3}$ & $\mathbf{4}$ & $\mathbf{5}$ & $\mathbf{6}$ & $\mathbf{7}$ & $\mathbf{8}$ & $\mathbf{9}$ & $\mathbf{1 0}$ \\
\hline CE-1 & 1.223 & 0.820 & 0.706 & 0.742 & 0.688 & 0.721 & 0.710 & 0.699 & 0.733 & 0.729 \\
CE-2 & 0.584 & 0.595 & 0.569 & 0.535 & 0.567 & 0.600 & 0.589 & 0.566 & 0.590 & 0.553 \\
CE-3 & 1.134 & 1.135 & 0.983 & 0.714 & 0.767 & 0.776 & 0.768 & 0.852 & 0.742 & 0.754 \\
CE-4 & 0.788 & 0.604 & 0.621 & 0.592 & 0.588 & 0.596 & 0.574 & 0.641 & 0.583 & 0.609 \\
CE-5 & 0.665 & 0.675 & 0.638 & 0.624 & 0.647 & 0.651 & 0.667 & 0.639 & 0.657 & 0.691 \\
CE-6 & 1.177 & 0.844 & 0.576 & 0.576 & 0.595 & 0.565 & 0.540 & 0.588 & 0.580 & 0.560 \\
CE-7 & 1.162 & 1.116 & 0.886 & 0.573 & 0.610 & 0.569 & 0.567 & 0.662 & 0.642 & 0.666 \\
CE-8 & 0.788 & 0.609 & 0.609 & 0.574 & 0.605 & 0.588 & 0.576 & 0.585 & 0.575 & 0.546 \\
\hline
\end{tabular}


Table S9. Test Set R2 and RMSE of machine learning results for each model of lattice constant.

\begin{tabular}{ccccccccccc}
\hline $\mathbf{R}^{\mathbf{2}}$ & $\mathbf{1}$ & $\mathbf{2}$ & $\mathbf{3}$ & $\mathbf{4}$ & $\mathbf{5}$ & $\mathbf{6}$ & $\mathbf{7}$ & $\mathbf{8}$ & $\mathbf{9}$ & $\mathbf{1 0}$ \\
\hline $\mathbf{C E - 1}$ & 0.160 & 0.924 & 0.925 & 0.923 & 0.920 & 0.921 & 0.927 & 0.923 & 0.923 & 0.921 \\
$\mathbf{C E - 2}$ & 0.961 & 0.963 & 0.963 & 0.963 & 0.962 & 0.962 & 0.962 & 0.962 & 0.959 & 0.962 \\
$\mathbf{C E - 3}$ & 0.757 & 0.755 & 0.754 & 0.955 & 0.955 & 0.953 & 0.954 & 0.953 & 0.954 & 0.951 \\
$\mathbf{C E - 4}$ & 0.827 & 0.963 & 0.964 & 0.961 & 0.963 & 0.961 & 0.964 & 0.963 & 0.962 & 0.963 \\
$\mathbf{C E - 5}$ & 0.823 & 0.961 & 0.957 & 0.939 & 0.934 & 0.928 & 0.948 & 0.955 & 0.933 & 0.944 \\
$\mathbf{C E - 6}$ & 0.158 & 0.962 & 0.960 & 0.960 & 0.962 & 0.962 & 0.960 & 0.960 & 0.961 & 0.960 \\
CE-7 & 0.759 & 0.755 & 0.758 & 0.963 & 0.962 & 0.964 & 0.964 & 0.961 & 0.962 & 0.962 \\
$\mathbf{C E - 8}$ & 0.960 & 0.963 & 0.963 & 0.963 & 0.960 & 0.962 & 0.961 & 0.962 & 0.963 & 0.963 \\
\hline \hline RMSE & $\mathbf{1}$ & $\mathbf{2}$ & $\mathbf{3}$ & $\mathbf{4}$ & $\mathbf{5}$ & $\mathbf{6}$ & $\mathbf{7}$ & $\mathbf{8}$ & $\mathbf{9}$ & $\mathbf{1 0}$ \\
\hline CE-1 & 0.356 & 0.107 & 0.105 & 0.107 & 0.109 & 0.108 & 0.104 & 0.107 & 0.106 & 0.109 \\
$\mathbf{C E - 2}$ & 0.077 & 0.075 & 0.075 & 0.074 & 0.075 & 0.075 & 0.075 & 0.075 & 0.078 & 0.074 \\
CE-3 & 0.191 & 0.191 & 0.191 & 0.082 & 0.082 & 0.084 & 0.083 & 0.083 & 0.082 & 0.084 \\
CE-4 & 0.160 & 0.074 & 0.073 & 0.076 & 0.075 & 0.077 & 0.072 & 0.075 & 0.075 & 0.074 \\
CE-5 & 0.163 & 0.076 & 0.080 & 0.096 & 0.099 & 0.103 & 0.087 & 0.081 & 0.100 & 0.091 \\
CE-6 & 0.355 & 0.075 & 0.076 & 0.077 & 0.075 & 0.075 & 0.077 & 0.076 & 0.075 & 0.077 \\
CE-7 & 0.190 & 0.190 & 0.190 & 0.074 & 0.075 & 0.073 & 0.074 & 0.075 & 0.075 & 0.075 \\
CE-8 & 0.077 & 0.074 & 0.074 & 0.073 & 0.077 & 0.075 & 0.076 & 0.075 & 0.074 & 0.074 \\
\hline
\end{tabular}



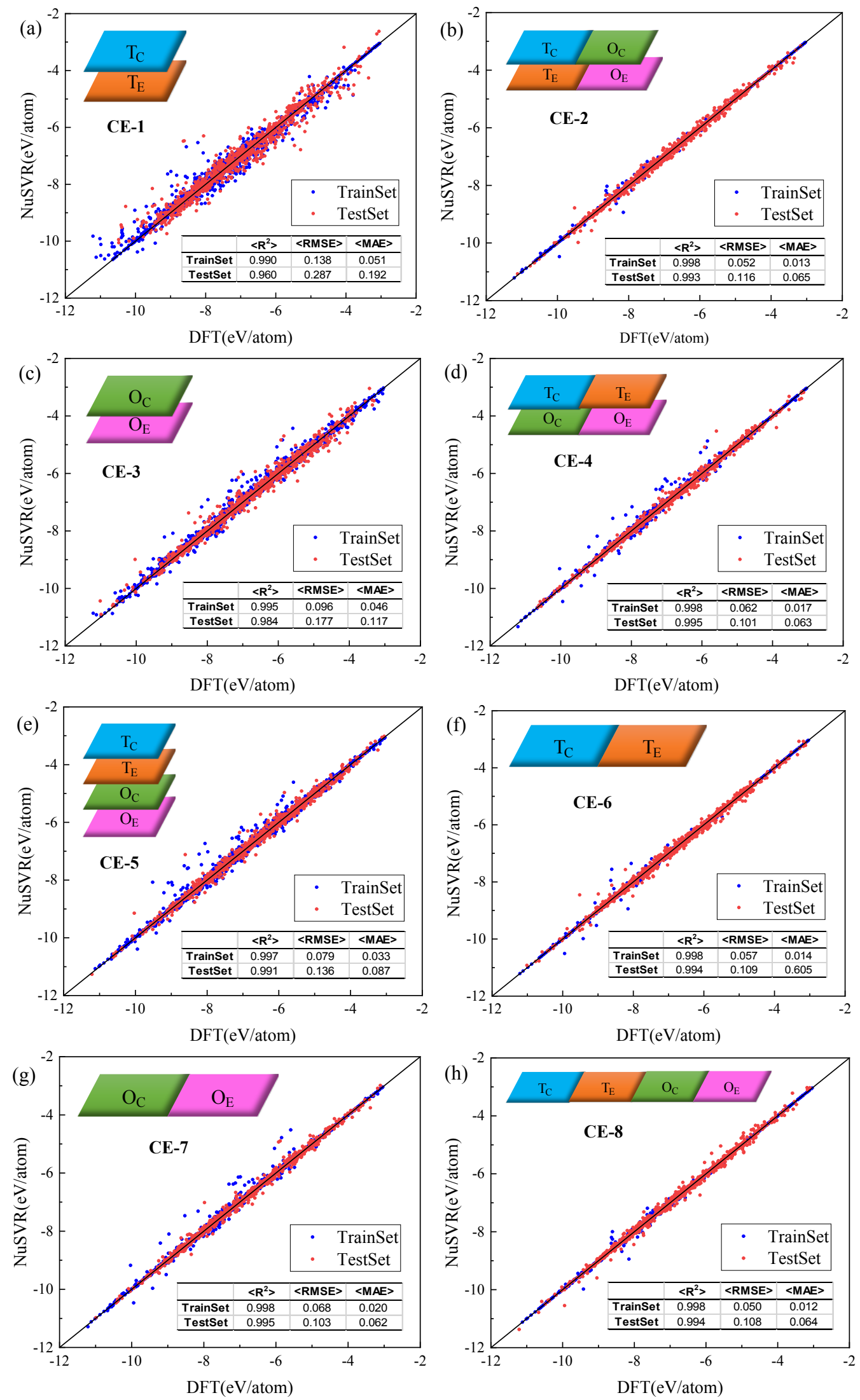

Figure S2. Comparison between the DFT calculations and machine learning predictions by SVR_rbf algorithm using CE-2 to CE-8 CE feature models at the nearest-neighbors, respectively, for the $[(a)$ to $(h)]$ total energy $(\mathrm{eV} /$ atom). 

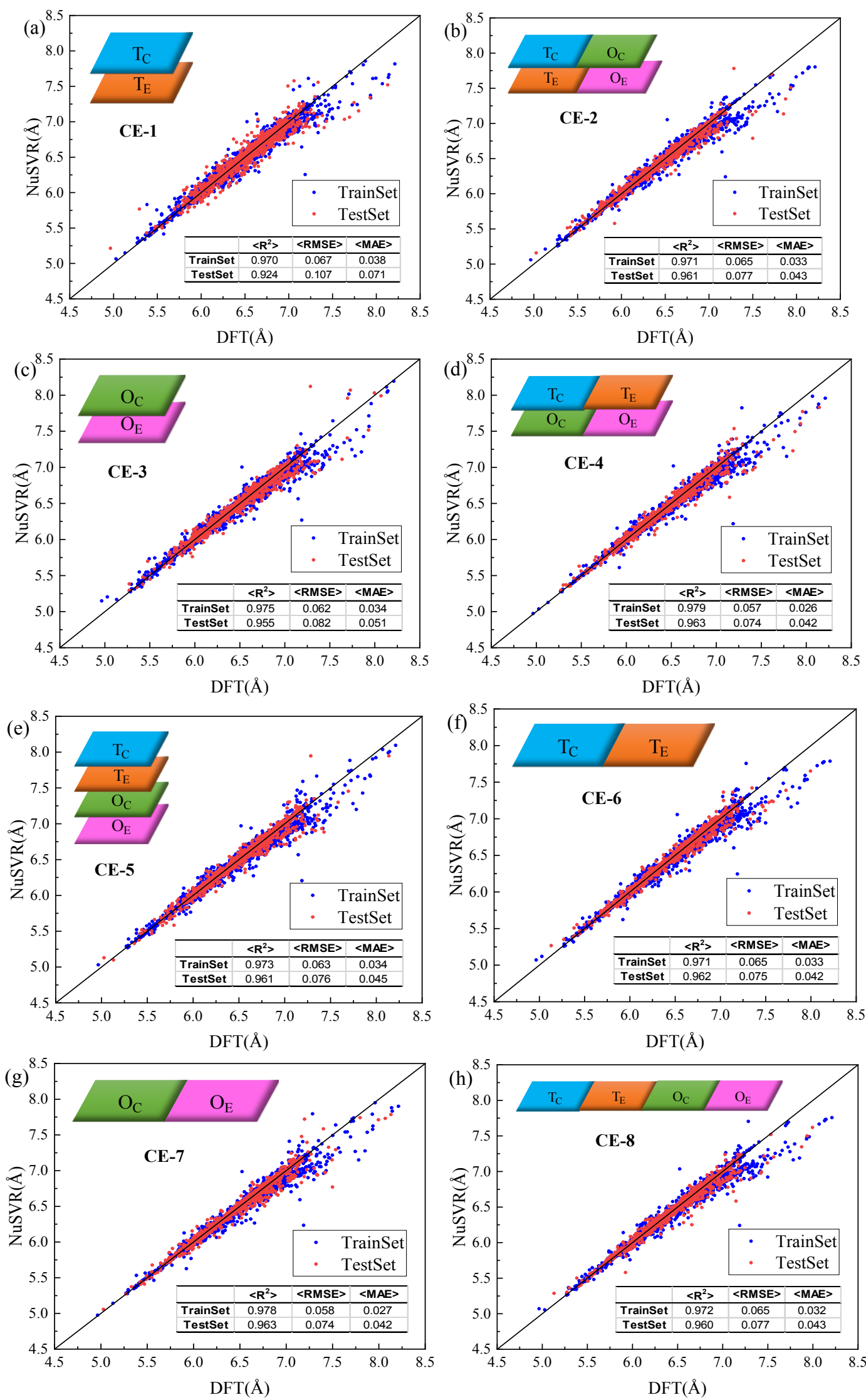

Figure S3. Comparison between the DFT calculations and machine learning predictions by SVR_rbf algorithm using CE-2 to CE-8 CE feature models at the nearest-neighbors, respectively, for the [(a) to (h)] lattice constant $(\AA)$. 


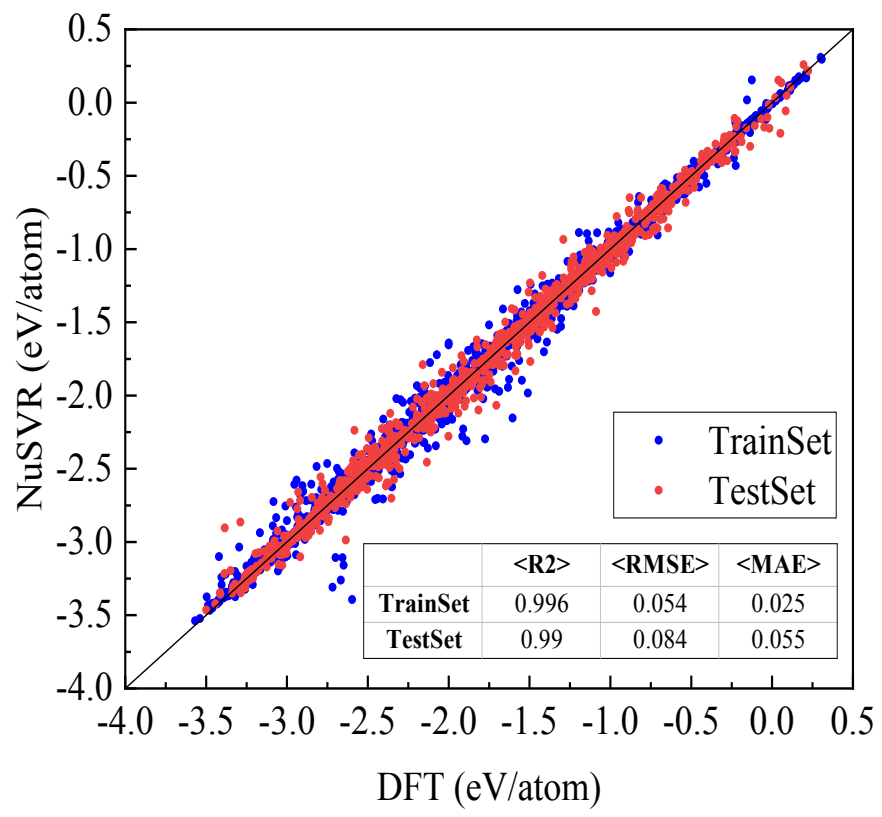

Figure S4. ML prediction results of formation energies (eV/atom) by composition models 